\title{
Workplace Stress among Nurses
}

\author{
Alqahtani Ibtesam Mohammed
}

King Saud University, School of Nursing, Riyadh 11451, Saudi Arabia

Corresponding author: Alqahtani Ibtesam Mohammed; Ibtesam.alqahtani@ mymail.barry.edu.

Received 27 November 2019;

Accepted 18 December 2019;

Published 20 December 2019

\begin{abstract}
Worldwide, occupational stress among care professionals, nurses in particular, is a major concern in health care systems. Work stress in nursing is linked to high rates of job dissatisfaction, burnout, absenteeism, turnover, and stress-related illness, thus placing job performance among nurses' and patients' lives at risk. The purpose of this integrative review is to explore the concepts of occupational stress among nurses. Three main theoretical models are included to illustrate different viewpoints of occupational stress. Meta-analysis of the basic literature and the results of previous research are used. Findings from studies have shown that evaluations of nursing work environments reflect a strong link with burnout. The excessive workload of nursing professionals, complexity of patient care activities, poor organized work environments, and lack of leaders' support are considered as contributing factor to the job stress which has resulted in providing unsafe care. The findings highlight that nurses frequently experience occupational stress due to the nature of the nursing profession. Thus, it is critical to implement effective organizational interventions to minimize work-related stressors and work performance of nurses. It is significant to have supportive working environments that encourage collaboration and empower nurses to provide excellent care and reduce work-related stressors.
\end{abstract}

Keywords: Stress, Workplace Stress, Nursing, Job Stress, and Occupational Stress.

\section{Background}

Stress is a universal phenomenon that affects everyone, specifically those in the healthcare professions. ${ }^{[4]}$ In the 17 th century, the term "stress" was used to describe suppression, sorrow, adversity, and discomfort, but the term changed in the 19th century to have the meaning of a strong influence exerted on a person or on a physical object. The word "stress" is derived from "stringere" which is the Latin word that means to "draw tight". ${ }^{[2,4]}$ Stress is defined as any experience that threatens people's health or adversely affects their normal functioning. ${ }^{[4]} \mathrm{A}$ state of experiencing stressors can be either harmful, which is known as distress, or beneficial and positive, which is known as eustress. ${ }^{[4]}$ Anyebe, Garba, Ukut, \& Hadiza (2014) cite Hans Selye, who defined stress in 1936 as a generalized, non-specific response of the body to any stimuli. This response may be physiology or psychology. The physiological response, known as the general adaptation syndrome (GAS), consists of three stages: alarm, resistance, and exhaustion. ${ }^{[4]}$ The psychological response is the individual reactions, either consciously or unconsciously, to the stressful events through the mediating process of cognition which includes interpretation, appraisal, reasoning, and coping. ${ }^{[4]}$

Yuwanich, Sandmark, \& Akhavan (2016) have stated that stress is as an environmental stimulus that affects an individual and results in physical and psychological reactions. People experience stress reactions when their coping strategies are unsuccessful. The work environment is considered a significant determinant of employee wellbeing. People who work in health care professions, in particular the nursing profession, are affected by their work environment and exposed to high levels of stress. ${ }^{[12]}$ Workplace stress, which can be also called as occupational stress or job stress, is defined as a harmful force that affects a person's well-being, either psychological or physical, and that occurs when job requirements do not match with the worker's needs, resources, and capabilities. ${ }^{[12]}$ Indeed, nursing is considered a highly stressful occupation due to the nature of the profession which requires nurses to confront severe illness, pain, violence, suffering, grief, and death. ${ }^{[12]}$

\section{Purpose}

The purpose of this integrative review is to expand the understanding of the concept of stress in the workplace, the main theoretical models of workplace stress, its effects on nurses, the nursing research that addresses this issue, and recommendations for future research.

\section{Theoretical Perspective}

There are many different theories of workplace stress that are significant to guide practice. A selection of key frameworks will be outlined below, including three major models to illustrate different viewpoints of occupational stress.

\section{Transactional Theories}

Richard Lazarus, who was a social-personality psychologist, developed a transactional theory of stress in 1966 to explain the dynamics of troublesome experiences. ${ }^{[2,11]}$ Transactional theories interpret stress as a direct product of a transaction or interaction between the environment and the person experiencing a situation. ${ }^{[2]}$ According to Lazarus, people interpret stress either as a stimulus or 
a response due to their differences. He points out that in previous theories there was a lack of explanation of the factors that help some people manage stress properly and others cannot cope with stressful stimuli. ${ }^{[2,11]}$ Thus, in transactional theories, environmental conditions are acknowledged as causes of stress for many individuals. The Lazarus model also takes into account cognitive processes and the factors affecting these processes. Stress, according to this theory, is an interactive process among three elements: the individual, his internal and external environment, and an individual's cognitive evaluation of environmental demands and stimulus response options. ${ }^{[2]}$ Daily, there is an amount of external and internal information that has been applied to the neurocognitive level of the body that is interpreted by the process of cognitive evaluation. ${ }^{[2]}$ Cognitive evaluation is the process of judgment by recognizing the methods, resources, and options that each person has to negotiate with potential or actual demands. ${ }^{[2]}$ Upon the process of cognitive evaluation, some people can assess events as positive, and others as very stressful. Intrinsic factors (intensity of external sources) and factors that are directly related to the individual (his beliefs) can influence the evaluation of the situation. ${ }^{[2,11]}$

\section{Job Characteristics Theory (JCT)}

The Job Characteristics Theory developed by Hackman and Oldham in 1976 proposes that negative or positive work characteristics result in mental states which lead to cognitive and behavioral outcomes such as satisfaction. ${ }^{[8]}$ In this model the five significant aspects of job characteristics include identity, skill variety, autonomy, task significance, and feedback. ${ }^{[8]}$ These characteristics lead to three critical psychological states which include experienced meaningfulness of work, experienced responsibility for outcomes, and knowledge of results. ${ }^{[8]}$ These psychological states, in turn, lead to achieving the following outcomes: high internal work motivation, high growth satisfaction, high general job satisfaction, and high work effectiveness. ${ }^{[8]}$

\section{Stimulus Based Models}

In 1967, psychologists Holmes and Rahe proposed the stimulusbased theory of stress to understand what happens when a person experiences changes in his life circumstances. This model views stress as a stimulus that causes certain reactions. ${ }^{[2]}$ According to this theory, any significant life changes or events may awaken normal, physiological, or psychological reactions, and as a result of that, risk of individuals to illness may increase. ${ }^{[2]}$ According to Holmes and Rahe, life events, either positive or negative, are considered stressful experiences for individuals and have normative or catastrophic impact. ${ }^{[2]}$ Holmes and Rahe developed the Social Readjustment Rating Scale (SRRS) to record the recent stressful experience of the individual, such as divorce. This scale indicates that people with higher scores in the SRRS are more likely to experience physical or mental illness. ${ }^{[2]}$ It is recommended to use this scale with caution, because the degree of stress that events cause is highly individual.

All the previously mentioned are main theoretical approaches for stress. Each one represents a key aspect of the stress process. These models acknowledge the significant role played by occupational environment in an individual's health. They are helping to identify distressing factors (stressors) in individuals' workplaces, understand subjective perceptions of people toward situations, and recognize people's differences in order to predict the possibility of individuals' adaptation to stressful situations. These theories are significant in guiding research related to occupational stress among nurses and in improving nursing practice through describing the ways nurses function in hospital settings and explain how the nature of nursing work may be implicated in many stressrelated physical and psychological problems which may then affect their professional functioning.

\section{Material and Method}

In this article use the method of meta-analysis of the basic literature and the results of previous research. ProQuest Central, PubMed, The Cumulative Index of Nursing and Allied Health Literature (CINAHL) Complete, and PsycINFO were accessed, and articles from the English-language literature published from 2014 through 2019 were obtained. Keywords included stress, workplace stress, nursing, job stress, and occupational stress. Inclusion of articles in the review was based on relevancy of content regarding background, definition, causes, coping, and consequences of workplace stress.

\section{Discussion}

\section{The Concepts of Job Stress}

A cross-sectional study has been done by Saquib, Zaghloul, Saquib, Alhomaidan, AlMohaimeed, and AlMazrou between January 2017 and June 2017. The study is conducted to assess the prevalence of stress, depression, and anxiety among 977 expatriate nurses in Saudi Arabia, and to examine their satisfaction with salary, workload, and teamwork. ${ }^{[10]}$ The data is collected by using the Depression Anxiety Stress Scales (DASS-21). Obtained data is analyzed with the Statistical Package for the Social Sciences (SPSS) software v25 and a two-sided test is used with alpha 0.05 . The majority of participants are females (99\%); the majority are from India or Pakistan $(60 \%)$, and $21 \%$ of them have worked in Saudi Arabia for more than 10 years. ${ }^{[10]} 70 \%$ of the participants are working in inpatient departments, while $23 \%$ are in emergency departments; $54 \%$ are working at least 9-10 hours per day (54\%); $47 \%$ of the nurses have between nine and 13 night shifts per month. ${ }^{[10]}$ Results from this study show that a significant portion of the expatriate nurses has symptoms of severe anxiety (33\%), depression (13\%), and stress $(11 \%) .{ }^{[10]}$ The findings also validate that $36.4 \%$ of those nurses are dissatisfied with their job workload, $29.4 \%$ are dissatisfied with salary, and $12.0 \%$ are dissatisfied with teamwork. Another significant finding of the study is that there is a significant correlation between workload and depression, anxiety and stress, while teamwork is significantly associated with depression only, and salary is not associated with any outcomes. ${ }^{[10]}$ The findings highlight that it is important to pay more attention to job-related factors that may affect the psychological well-being of nurses. Reducing workload and improving communication between nurses and other health care providers are important to increase the positive feelings of nurses towards the workplace, enhance job satisfaction, promote retention, and prevent burnout among nurses.

Another cross-sectional study has been conducted to compare perceptions of occupational stress among 2,616 shift charge nurses in three countries, which include Israel, the USA (state of Ohio), and Thailand. ${ }^{[1]}$ The charge Nurse Stress Questionnaire (CNSQ) is used to collect the data and the obtained data is analyzed with the Statistical Package for the Social Sciences (SPSS) software v21. ${ }^{[1]}$ Results show that participants are predominantly female (93.2\%) and $69.8 \%$ of them are registered nurses. ${ }^{[1]}$ Most of these charge nurses work full time $(88.6 \%)$, and $39.3 \%$ of them have worked as nurses for 15 years or more. ${ }^{[1]}$ The study highlights that the level of stress is moderate among the total sample (2.84 on a scale of 1-5). The highest overall stress level is among the Thai charge nurses and the lowest is among Israeli charge nurses. ${ }^{[1]}$ Study reveals that 
the highest significant stress level is found among young charge nurses, under the age of 30 years, with fewer years of work experience or experience as charge nurses in all three countries. ${ }^{[1]}$ Across the three countries, nurses considered the "responsibility burden and lack of resources" as the leading most stressful factor, while the "patient and family complaints" is the least stressful factor. ${ }^{[1]}$ The findings highlight that the role of shift charge nurses is complex. Reducing work-related stress among shift charge nurses is crucial for effective management of their duties in wards, which directly impacts on the quality and safety of patient care.

De Almeida, Shadvar, Lepage, \& Rennick (2016) conduct a qualitative study in Canada to explore the perceptions of pediatric nurses toward work-related stressors in medical and surgical units. 65 nurses are eligible to participate in the study and data collected until data saturation is reached at 12 . The study reveals that the majority of participants who are providing care for sick childern describe a strong sense of responsibility for providing excellent care. ${ }^{[3]}$ The study reveals also that nurses feel powerlessness to provide quality care when confronted with limited resources and lack of support. ${ }^{[3]}$ Caring for children who are very sick is also identified as a major source of work-related stress. Participants describe the feeling of pressure and overwhelmed due to the role and multiple responsibilities they carried on their shoulders. ${ }^{[3]}$ The findings highlight that pediatric nurses who are working on units that provide care for different medical and surgical services experience physical and mental exhaustion due to the fact that they are unable to take breaks, leave work on time, and forget to take care of themselves. ${ }^{[3]}$ Building supportive working environments that encourage collaboration and empower nurses is important to provide excellent care and reduce work-related stressors.

Another qualitative study has been conducted by Yuwanich, Sandmark, \& Akhavan in 2016 to explore the perceptions of nurses toward occupational stress in an emergency department (ED) in Bangkok, Thailand. The participants in this study are 21 ED nurses; 17 are female and 4 are male. The findings of this study reveal that the nurses perceive heavy work-load related to their responsibilities and tasks as a significant stressor in the workplace. ${ }^{[12]}$ The findings also show that the nurses are unable to complete all of their caring tasks due to limited work time, which is considered a significant stressor in the workplace. Another powerful source of stress in the workplace is that there is a shortage in the number of physicians in the hospital and that leads nurses to take on the physician's role to save patients' lives. ${ }^{[12]}$ This finding supports that shortage of nursing staff, conflict between the nurses and physicians, low income, and insufficient support from supervisors are perceived as stressors. ${ }^{[12]}$ Consequently, occupational stress in the ED has adverse impact on participants' physiological and psychological health, and the quality of nursing care becomes below-standard. ${ }^{[12]}$ The findings highlight that nurses frequently experience occupational stress due to the nature of the nursing profession. ${ }^{[12]}$ Thus, it is critical to implement effective organizational interventions to minimize work-related stressors, improve efficiency and effectiveness in nursing care, and promote retention.

\section{The Concepts of Job Stress Related to Burnout}

There is a growing concern related to the effects of job stress on nursing professionals. Nurses represent the most numerous groups of health professionals who provide patient care 24 hours per day. ${ }^{[7]}$ The work conditions in nursing produce a state of chronic stress due to work overload, inadequate staffing, advanced technology, lack of motivation, and complexity of cases. Thus, the consequences of occupational stress can lead to poor physical, social, and psychological health for nurses and can also negatively impact patient safety and the quality of care. ${ }^{[7]} \mathrm{A}$ substantial literature supports the view that nurses deal with high levels of stress in their work environment, and as a consequence of that, they experience a high prevalence of psychological problems that undermine their well-being. According to Donnelly (2014), workplace stress is a major contributing factor, and by 2020 , the size of the registered nurse workforce is forecast to be $20 \%$ below the healthcare systems' requirements worldwide. Evidence shows that prolonged exposure to stressful working environments in nursing is associated with high turnover, sickness, absenteeism, poor job satisfaction, psychologic burnout, and decreased quality of care. $^{[4,5,6,9]}$

Burnout is a growing occupational health problem that has been linked to prolonged exposure to professional stress. Psychological burnout is characterized by high level of physical, emotional, and spiritual exhaustion. The term "burnout" has been described as feelings of energy depletion, depersonalization, and negativism toward one's job, and the sense of reduced personal accomplishment in the "helping" professions. Psychological burnout represents individuals' internal response to stressors in occupational environments. A cross-sectional study in a sample of 258 baccalaureate and auxiliary nurses at a tertiary hospital at the Public Health System of Andalucía found that the level of emotional exhaustion among participants was medium, the level of depersonalization was high, and the level of personal accomplishment was low. ${ }^{[9]}$ The study revealed also that there is a significant and positive relationship between work-related stress and emotional exhaustion and depersonalization. ${ }^{[9]}$ Another aspect that emerged in the results was that having excessive workload and having trainees under one's supervision are factors for professional burnout. ${ }^{[9]}$ Filgueira, Pereira, \& Sousa (2017) cited another study that was conducted in the Northeast of Brazil to assess the dimensions of burnout among nursing professionals. The study revealed that $68.3 \%$ of the participants present at least one dimension of burnout syndrome and the level of emotional exhaustion and depersonalization, which are significantly high due to work-related stress. ${ }^{[5]}$

The consequences of professional burnout are increased risk of mental fatigue, increased risk of cardiovascular diseases, reduced levels of self-motivation, and increased rates of absenteeism. ${ }^{[9]}$ Yuwanich, Sandmark, \& Akhavan (2016) state that work-related stress and burnout may lead to reduced staff productivity by affecting their ability to perform their roles effectively, produce confusion and irritability, and promote negative feelings such as anger toward themselves. More conflicts, disagreements, job dissatisfaction, and retention may also occur among nursing staff as a result of job stress and burnout. ${ }^{[12]}$ These consequences place patients' lives at risk. ${ }^{[5]}$ Thus, nursing staff needs to have good psychological health to provide safe, efficient, and effective care to patients.

\section{Conclusion}

In the hospital context of nursing professionals, this review provides valuable insight into the complexity of work as a nurse and enables the managers of healthcare organizations to better understand the relations between occupational stressors, burnout syndrome, and patients' safety. According to the analyzed studies, the excessive workload of nursing professionals, complexity of patient care activities, poor organized work environments, and lack of leaders' support are considered contributing factors to job stress, dissatisfaction, and burnout which result in unsafe care. Addressing 
the issues that surround nursing practice environments can impact the healthcare system through improving the quality of care delivered to patients and improving the retention of highly skilled nurses. Nurse managers, in particular, play a significant role to manage frustration and stress among subordinates.

\section{Recommendations}

Given the lack of current research into occupational stressors, further research is clearly warranted. Relationships between staff nurses and other health care professionals are particularly important when examining occupational stress. In addition, conducting qualitative research in numerous countries would enable a greater depth of understanding for the determination of job stress factors and for the contributions of stressful work environments on nurses' and patients' wellbeing, organizational outcomes, and health care costs.

\section{References}

[1] Admi, H., \& Eilon-Moshe, Y. (2016). Do hospital shift charge nurses from different cultures experience similar stress? An international cross-sectional study. International Journal of Nursing Studies, 63, 48-57. https://doi.org/10.1016/j.ijnurstu.2016.08.005

[2] Anyebe, E. E., Garba, S. N., Ukut, C. I., \& Hadiza, L. (2014). An assessment of stress amongst nurses in zaria metropolis, northwest nigeria. West African Journal of Nursing, 25(1), 53-70. Retrieved from https://searchebscohost-

com.ezproxy.barry.edu/login.aspx?direct $=$ true $\& \mathrm{db}=\mathrm{ccm}$ $\& \mathrm{AN}=107773560 \&$ site $=$ ehost-live

[3] De Almeida, V, A., Shadvar, S., Lepage, S., \& Rennick, J. E. (2016). Experienced pediatric nurses' perceptions of work-related stressors on general medical and surgical units: A qualitative study. International Journal of Nursing Studies, 60, 216-224. https://doi.org/10.1016/j.ijnurstu.2016.05.005

[4] Donnelly, T. (2014). Stress among nurses working in an acute hospital in Ireland. British Journal of Nursing, 23(13), 746-750. https://doi.org/10.12968/bjon.2014.23.13.746

[5] Filgueira, C. C., Pereira, V. E., \& Sousa, P. (2017). Patient safety and nursing: interface with stress and Burnout Syndrome. Revista Brasileira de Enfermagem, 70(5), 1083-1088. https://doi.org/10.1590/0034-71672016-0194

[6] Hauge, L.J., Skogstad, A., \& Einarsen, S. (2007). Relationships between stressful work environments and bullying: results of a large representative study. Work \& Stress, 21(3), 220-242. Retrieved from https://searchebscohost-

com.ezproxy.barry.edu/login.aspx?direct $=$ true $\& d b=c \mathrm{~cm}$ $\& \mathrm{AN}=105876646 \&$ site $=$ ehost-live

[7] Munnangi, S., Dupiton, L., Boutin, A., \& Angus, L. D. G. (2018). Burnout, perceived stress, and job satisfaction among trauma nurses at a level I safety-net trauma center. Journal of Trauma Nursing, 25(1), 4-13. https://doi-

org.ezproxy.uakron.edu:2443/10.1097/JTN.00000000000 00335

[8] Oerlemans, W. G. M., \& Bakker, A. B. (2018). Motivating job characteristics and happiness at work: A multilevel perspective. Journal of Applied Psychology, 103(11), 1230-1241. https://doi.org/10.1037/ap10000318

[9] Portero, S., \& Vaquero, M. (2015). Professional burnout, stress and job satisfaction of nursing staff at a university hospital. Revista Latino-Americana de Enfermagem (RLAE), 23(3), 543-552. https://doi.org/10.1590/01041169.0284.2586

[10] Saquib, N., Zaghloul, M., Saquib, J., Alhomaidan, H., AlMohaimeed, M., \& AlMazrou, M. (2019). Association of cumulative job dissatisfaction with depression, anxiety and stress among expatriate nurses in Saudi Arabia. Journal of Nursing Management (John Wiley \& Sons, Inc.), 27(4), 740-748. https://doiorg.ezproxy.uakron.edu:2443/10.1111/jonm.12 762

[11] Spangenberg, J. J., \& de Villiers, A. (2017). Stress and its implications for family practice. Professional Nursing Today, 21(4), 24-28. Retrieved from https://search.ebscohost.com/login.aspx?direct=true \&db= ccm\&AN=130020131\&site=ehost-live

[12] Yuwanich, N., Sandmark, H., \& Akhavan, S. (2016). Emergency department nurses' experiences of occupational stress: A qualitative study from a public hospital in Bangkok, Thailand. Work, 53(4), 885-897. https://doi.org/10.3233/WOR-152181

\section{Author Profile}

Alqahtani Ibtesam received a B.S. degree in Nursing from King Saud University in the Kingdom of Saudi Arabia, and received an M.S degree in Nursing Education from Roberts Wesleyan College in the United States of America respectively. Currently, she is completing her $\mathrm{PhD}$ in Nursing at Barry University in the state of Florida, the United States of America. 